Editorial Manager(tm) for Spine Journal Manuscript Draft

Manuscript Number: SPINE F11111 Title: Anterior approach to cervical spine Article Type: Focus Paper Keywords: Cervical Spine; surgery Corresponding Author: Kenneth MC. Cheung, FRCS, FHKCOS, FHKAM(Orth) Corresponding Author's Institution: The University of Hong Kong Medical Centre First Author: Kenneth MC. Cheung, FRCS, FHKCOS, FHKAM(Orth) Order of Authors: Kenneth MC. Cheung, FRCS, FHKCOS, FHKAM(Orth);K.C. Mak, FRCS(Ed),

FHKAM(Orth),;Keith D.K. Luk, MCh(Ortho), FRCSE, FRCSG, FRACS, FHKAM (Ortho) 


\section{Anterior approach to cervical spine}


Running Head: Anterior approach to cervical spine

Order of Authors: Kenneth M C Cheung, MD, FRCS, FHKAM(Orth),

K C Mak, FRCS(Ed), FHKAM(Orth),

Keith D K Luk, MCh(Ortho), FRCSE, FRCSG, FRACS, FHKAM (Ortho)

Keywords: $\quad$ anterior cervical decompression; transoral approach; anterolateral approach; manubriotomy

Correspondence: ～Kenneth M.C. Cheung, MBBS, MD, FRCS, FHKCOS, FHKAM(Orth) Department of Orthopaedics \& Traumatology Queen Mary Hospital

102 Pokfulam Road

Hong Kong, SAR, China

Tel: +85222554254

Fax: +852 28174392

Email: ken-cheung@hku.hk

Affiliations: Department of Orthopaedics and Traumatology, The University of Hong Kong, Pokfulam, Hong Kong SAR, China

Disclosure: $\quad$ The authors have no financial or competing interests to disclose. 


\begin{abstract}
Study Design: Review of surgical technique

Objective: To provide accounts of the authors’ preferred methods for performing anterior cervical surgery with personal tips and pearls.
\end{abstract}

Summary of Background Data: Many have described the various anterior surgical approaches to the cervical spine, and in this review we hope to describe our preferences, highlighted with some tips and pearls.

Methods: Various accounts of the transoral, the anterolateral (Smith-Robinson) and the split manubrium approaches were reviewed and used as the basis of the review. Additional notes with regards to the authors' preference were noted to provide further guidance. The description were delineated from the most cephalad to the most caudal.

Results: The transoral, the anterolateral (Smith-Robinson) approach and the manubriotomy approach were described. Each account starts with the basic pre-operative considerations, then describes the incision and the main anatomical landmarks, and finally concludes with closure and main complications to monitor for. A brief description of the main pathologies that each approach may address is also provided.

Conclusion: The 3 anterior approaches to the cervical spine are direct and elegant solutions to pathologies arising from the anterior column. They supplement the more commonly used posterior approaches, which provide stronger multilevel fixation, and thus is an essential tool in the armamentarium of a spine surgeon. 


\section{Key Points}

1 The transoral approach provides access to $\mathrm{C} 0$ to $\mathrm{C} 2$ with prerequisites including adequate interdental distance, neck hyperextension, and specialized retractors.

2 Once the anterior tubercle of C1 is identified for the incision of the transoral approach, the main caution is not to go wider than $15 \mathrm{~mm}$ from the midline least the vertebral artery is injured.

3 The anterolateral (Smith-Robinson) approach is the most commonly employed of the three and allows access from C3 to cervicothoracic junction.

4 We prefer to approach from the right side for C3 to C6, and from the left for cervicothoracic junction to avoid need to protect the recurrent laryngeal nerve.

5 The manubriotomy approach can either be 1-or 2-sided thereby affording customized access as far down as T4, without the morbidity of a full sternal split or clavicular osteotomies. 
*Mini Abstract (50 words)

\section{Mini-abstract}

The classic transoral and anterolateral (Smith-Robinson) approaches which allow direct anterior access to the cervical spine is described with the authors' preferences highlighted. The 1-or 2sided manubriotomy provides an approach to the cervicothoracic junction without the morbidity of the full sternotomy. 


\section{Introduction}

The anterior approach to the cervical spine is an extremely practical and elegant approach to dealing with pathologies that arise primarily from the anterior spinal column. Well known surgeons have described their approach to the anterior cervical spine in previous publications, ${ }^{1-3}$ and in this article we hope to provide some notes on our preferred approach and some tips and pearls along the way. The approaches to the anterior cervical spine may be divided into three for convenience of description. They are the transoral, the anterolateral cervical, and the split manubrium approaches. The well-established transoral approach affords a reasonably wide runway to $\mathrm{C} 1$ and $\mathrm{C} 2$. And the more recently proposed split manubrium approach ${ }^{4}$ allows access to the lowermost cervical and upper thoracic levels, down to T4, without having excess morbidity from damaging the sternum caudally.

Differing pathologies require different methods to address them, and the anterior approaches compliment that of the posterior to allow the surgeon to attain the treatment objectives without compromise. We hope that readers could gain a utilitarian understanding of managing diseases ranging from the craniocervical to the cervicothoracic junction, and could start making good use of them to help suitable patients. Our description of the methods will be from cephalad to caudal.

\section{Transoral approach}

\section{Pre-operative considerations}

The transoral approach affords a direct working portal to the anterior aspects of C0 to C3 (Figure 1). And despite having to cut through a number of layers, including mucosa, fascia and the median raphe of the prevertebral muscles, the cut is relatively thin and avascular. ${ }^{1}$

The approach is dictated by the trajectory through the oral opening, and this in turn is determined by neck extension and interdental distance. The latter is recommended to be at least $25 \mathrm{~mm}$ to allow a reasonable working portal. ${ }^{5}$ Temporomandibular joint stiffness may 
reduce the interdental distance, while subaxial cervical kyphosis and basilar invagination may alter the trajectory as well. ${ }^{6}$ A Mayfield retractor with $2-3 \mathrm{~kg}$ traction and a roll under the scapulae is helpful in ensuring hyperextension of the cervical spine and stabilizing the head and neck. One of the most useful preoperative assessments is the lateral radiograph taken with the neck in hyperextension. It also allows screening for any neurological complaints with that position.

Prophylactic antibiotic coverage is with broad spectrum coverage for both gram positive and gram negative organisms. ${ }^{7}$ Preoperative culture swabs are taken least there should be an infection and require specific antibiotic therapy.

\section{Airway protection and incision}

Airway protection is especially important for this procedure. Orotracheal intubation is our preferred approach. Alternatives include nasotracheal intubation or tracheostomy. The latter is indicated in those who require ventilation for a prolonged period after the surgery. ${ }^{8}$ Specialized retractors, e.g. Davis-Crow or Spetzler-Sonntag transoral retractors, are used to maintain the width of the exposure. A self-retaining malleable retractor is used to elevate the soft palate. If this is not adequate then the soft palate may be split in the midline and repaired en masse later. And if a more cephalad trajectory is required, the posterior portion of the hard palate may be removed and stabilized with miniplates afterward. ${ }^{7}$ Alternatively, a midline posterior split of the hard palate with infracture into the nasopharynx and removal of the vomer may be used. ${ }^{9}$ Care should be taken not to cause excessive compression on the tongue or lips. And finally, a throact pack is used to prevent debris from falling into the airway and the patient is placed in a Trendelenburg position to reduce the chance of aspiration. ${ }^{10}$

Before making the incision, a lateral x-ray is suggested to confirm the final alignment and position of the palate, the mandible and the spine. The key landmark to palpate and identify is the anterior tubercle of $\mathrm{C} 1$ (Figure 2), which is the most prominent point and normally should lie in the midline. This prominence is particularly evident in those with atlantoaxial dislocation. ${ }^{1}$ A patient with $\mathrm{C} 1 / 2$ rotatory subluxation may have the lateral mass rotated toward the midline and thus lead to disastrous violation of the vertebral artery. ${ }^{6}$ 
The incision should be directly down to bone, ${ }^{1}$ with an attempt to preserve the pharyngomucosal flap for subsequent closure. ${ }^{8}$ With the center of the incision about $1 \mathrm{~cm}$ caudal to the anterior tubercle, a $3-5 \mathrm{~cm}$ longitudinal cut is made. ${ }^{1}$ Subperiosteal dissection is performed as far laterally as the lateral margin of the lateral masses, ${ }^{1}$ therefore also raising the anterior longitudinal ligament and longus colli muscles. Closure should be done in layers with anterior longitudinal ligament and longus colli muscles in the deeper layers, and the overlying layer of fascia and mucosa sutured as one layer. ${ }^{6}$

\section{Resections}

Lateral lying structures include the vertebral arteries, eustachian tubes and hypoglossal nerves. Bony removal of the anterior arch of $\mathrm{C} 1$ should not extend beyond more than 15 $\mathrm{mm}$ from the midline to avoid injuring the adjacent structures. ${ }^{78}$ Safety margins lies within $11 \mathrm{~mm}$ from the midline at the foramen magnum, $24 \mathrm{~mm}$ at the atlas and $14 \mathrm{~mm}$ at the axis lower border. ${ }^{7}$

Resection of the dens is performed in stages with initial thinning of the cortex using a high speed burr, and final removal either by burr, or a $1 \mathrm{~mm}$ Kerrison ronguer, or an angled curette (Figure 3). However, final resection of the dens cortical shell should be performed after removal of the alar and apical ligaments to ensure tension in the ligaments for ease of resection. Great care is needed for removal of the dens cortical shell as there may be adhesions to the dura for inflammatory entities. Finally, the transverse ligaments should be preserved to ensure lateral stability of $\mathrm{C} 1$ on $\mathrm{C} 2 .{ }^{6}$ And since the transoral procedure often results in destabilization, a posterior fusion is necessary. We prefer immediate posterior stabilization, and therefore in using the Jackson table. Though sometimes, it is only with the post-operative flexion-extension radiographs that the instability is noted.

\section{Post-operative care and complications}

The endotracheal tube is may be retained for 24 hours in view of the risk for tongue swelling, though local application of steroid cream may help reduce this post-operative swelling. A Ryle's tube is inserted to continue drainage of the gastric contents and prevent reflux, and kept for 5 - 7 days. After which clear fluids may be allowed and regular diet 
resumed by around day 14. And to prevent wound infection, antibiotics is usually continued for 3 days after the operation.

Post-operative wound breakdown or infection is of primary concern. Wound inspection, especially for the cephalad portion, often requires use of endoscope through the nasal passage. Early wound dehiscence is usually due to poor re-apposition. ${ }^{6}$ But retropharyngeal abscesses usually develop later, after 5 - 7 days, and require surgical drainage.

The other main complication of the transoral procedure is CSF leakage. ${ }^{6}$ If there was suspicion of a dural tear, checking for CSF leakage by way of the Valsalva maneuver may be necessary. Dural tear may be reapproximated or repaired using a fascial graft. Fibrin glue is often used for additional security. In case of an established CSF fistula, insert a lumbar drain and give antibiotic coverage for the duration that the drain is in situ.

The transoral approach is a very useful and direct approach to pathologies located in the upper (C0-C3) anterior cervical spine, though it may entail extra caution by way of the structures lying lateral to the vertebral column and possibly closely adherent dura mater posteriorly.

\section{Anterolateral (Smith-Robinson) approach}

This is probably the most widely used approach to the cervical that was first described by Robinson \& Smith ${ }^{11}$ and later modified by Southwick \& Robinson ${ }^{3}$ to access C3 down to T1. Thus this account it not meant to be exhaustive, but includes some personal preferences and tips for the readers' cosideration.

\section{Pre-operative considerations}

The patient is positioned in the supine position with the head slightly rotated to the left, and with a rolled towel or equivalent placed transversely under the scapulae to allow the 
neck to slightly extend. With this position, the chin-chest distance is increased allowing a larger working area, and also opens up the intervertebral disc spaces anteriorly facilitating discectomies. If iliac crest bone graft is needed, we place another roll under the iliac wing on the side of graft harvest to facilitate surgical exposure. Contrary to descriptions in the literature, ${ }^{12}$ our preference is to approach the spine from the right side from C3 to C6, while for the cervicothroacic junction to approach from the left side to avoid the need to expose and protect the recurrent laryngeal nerve. ${ }^{13}$ This preference is based on the authors being right-handed, and thus an approach from the right side, favoring the right hand is preferred. Of course, other considerations such as a local injury or infection or prior surgery, e.g. radical neck dissection, may preclude from choosing the desired side of approach. Intraoperative traction by use of Garner-Wells tongs or Mayfield clamps are rarely used or needed except in cases where some deformity correction is needed. Usually the neck and head is stabilized by a well-padded head cushion.

\section{Skin incision \& dissection}

A transverse incision along the skin crease of some $3-5 \mathrm{~cm}$ in length, is adequate for the exposure of 2 to 3-disc levels, while an extensile longitudinal incision parallel and anterior to the sternocleidomastoid muscle is used for more extensive exposures ${ }^{13}$ (Figure 4). The level of incision can be determined by using surface landmarks, but more commonly with pre-incision radiologic screening and skin marking, as this allows a more accurate determination of levels and therefore the size of the incision needed. The incision should be at the mid-point of the level of surgery or just cephalad since retraction caudally is easier. ${ }^{14}$ The approach utilizes internervous planes between the sternocleidomastoid and the longus colli muscles (Figure 5).

Once past the skin, the platysma may be split in the same direction as the skin incision. Blunt dissection is used all the way to the vertebral body. Care is taken during the dissection to identify and either coagulate or ligate all bleeding vessels to ensure a clear operating field. Care is taken to ensure an adequate longitudinal and lateral release to allow clear visualization and adequate mobilization and protection of the trachea and esophagus 
together with the recurrent laryngeal nerve. The common carotid artery and internal jugular vein are routinely identified and protected by gentle lateral retraction.

Care should be taken when retracting the esophagus as intraoperative perforation and postoperative fistula are known rare complications. The middle thyroid veins may be ligated without adversely sequalae, but caution is advised when approaching more cephalad (i.e. up to C3), where there is the superior thyroid artery. ${ }^{13}$ The superior laryngeal nerve often is near the superior thyroid artery, and damage to this nerve will paralyze the ipsilateral vocal cord. The omohyoid may be encountered when approaching the lower cervical area and therefore be transected. The authors' preference for single level surgeries would be to preserve and retract it. Care is taken to extensively push the esophagus off the pre-vertebral fascia using blunt dissection, as this facilitates exposure and reduces the risk of damage to the esophagus.

It is important to identify both sides of the longus coli muscles, as this provides a excellent visual guide to the midline. The sympathetic trunk converge as it goes caudal while the longus colli muscle diverge. They intersect around the level of C6 near the anterior border of the longus colli, and care should be taken to avoid injuring it. ${ }^{15}$

Level confirmation should always be carried out using intraoperative imaging.

Once levels are confirmed, further mobilization would involve retraction of the longus coli muscles, and coagulation of the segmental vessels at the levels concerned. For a single level diskectomy, the authors' preference is to transversely cut part of the longus coli fibers to facilitate lateral exposure without the need to extensively elevate the muscle to allow adequate lateral retraction.

\section{Decompression}

The techniques of decompression would vary between surgeons but usually start with a diskectomy and resection of anterior osteophytes at the involved level. Some points are worthy of note. Firstly width of decompression is judged adequate when both uncovertebral joints can be seen. This is usually around $15 \mathrm{~mm}$ in width. The only caveat to dissecting till the joint of Luschka is when pre-operative CT shows an aberrantly medial 
vertebral artery in the mid-body of the vertebral body, which may be just medial (average $0.14 \mathrm{~mm}$ to be precise) to the uncovertebral joint. ${ }^{16}$ Secondly, the authors prefer to routinely remove the posterior annulus and posterior longitudinal ligament to expose the dura. This is to ensure that all disc fragments including possibily sequestrated fragments are removed. The greatest worry is violation of the vertebral arteries just lateral to the uncovertebral joints.

The mid-cervical anterolateral approach is the workhorse of anterior cervical surgical approaches. It represents a relatively atraumatic dissection to the mid-cervical elements with minimal blood loss, and can be used to tackle the majority of anterior spinal pathologies.

\section{Manubriotomy approach}

Depending on the position of the manubrial notch, the majority of the cervical pathologies can be accessed through the Southwick \& Robinson approach. However in cases where the notch is high or where the pathologies extend into the upper thoracic spine, then a more extensile approach maybe necessary. An easy way to tell would be to take a lateral view of the upper thoracic/lower cervical spine, and then extend a horizontal line from the upper border of the manubrial notch to the spine. Where the line crosses the spine would indicate the lower limit of a cervical exposure without splitting the manubrium. Usually this can be as low as T2, but may vary between individuals.

A number of different approaches to the cervicothoracic junctions have been described, 17-19 the authors's preference is to use a split manubrium approach, described by Luk et al., ${ }^{4}$ for its relative ease of exposure. Such an approach would allow further extension of the exposure to $\mathrm{T} 4$, while avoiding the morbidity of a full sternal split or clavicular osteotomies.

Pre-operative considerations 
The split manubrial approach has been described as either a L-shape split, in which the exposure width is approximately $4 \mathrm{~cm}$ or with a bilateral split as an inverted $\mathrm{T}$ which affords a $8 \mathrm{~cm}$ width of manubrial retraction. This approach can be combined with a Southwick-Robinson approach to allow for an extensile exposure from C3 down to T4. As with any anterior cervical procedure, good neck extension is essential. A rolled towel is placed under the scapulae to extend the neck and pull the shoulders back.

\section{Skin incision \& dissection}

For a detailed descriotion of the approach, the readers are referred to the original description by Luk et al. ${ }^{4}$ The incision starts from the manubrial notch and the caudal end needs only be $3 \mathrm{~cm}$ caudad to the manubriosternal angle (Figure 6). The cephalad arm may be extended as required to facilitate mid-cervical spine exposure. A $2 \mathrm{~cm}$ wide skin flap is raised from the manubriosternum bilaterally, and with finger dissection the posterior manubrial surface is freed. In younger patients, the thymus may be encountered. The internal thoracic artery (previously known as the internal mammary) is encountered and ligated around the second intercostal space, which is where the transverse limb of the osteotomy should exit. A unilateral transverse cut, or bilateral cuts can be made with an oscillating saw to achieve the width of exposure required (Figure 7). A retractor can then be placed vertically to maintain exposure to the anterior mediastinum and finger dissection used to mobilize the retrosternal soft tissue.

In the U-shaped pocket, the common carotid artery will border on the left while the brachiocephalic artery and vein will border on the right. The floor is formed by the trachea and esophagus and can be retracted left to expose the spine. Identification of the right recurrent laryngeal nerve is imperative at this stage. It loops under the right subclavian artery, a branch of the brachiocephalic trunk, and ascends near the trough formed by the trachea and esophagus on their right side.

With the above dissection, C7 to T4 could be approached comfortably. However, to approach T5, it may be necessary to dissect along the lateral border of the ascending aorta until the upper border of the heart. The left brachiocephalic vein may have to be retracted distally, and even ligated to ensure adequate exposure. 
This approach is relatively demanding and it is recommended that a cardiothoracic surgeon should be involved, at least, in the initial experience. A number of important sturctures need to be identified and protected and these are summarized below and in Figure 8:

1. Left: common carotid artery

2. Right: right brachiocephalic (innominate) artery and vein

3. Caudad: left brachiocephalic vein

4. "Floor": trachea and esophagus

5. Right recurrent laryngeal nerve - ascends between the trachea and esophagus

6. Thoracic duct - if approaching from left side of the esophagus

The manubriotomy, whether unilateral or bilateral, is a versatile and extensile approach to the cervicothoracic junction that allows exposure down to $\mathrm{T} 4$ with relative ease, and extension to the mid-cervical spine simple by way of the conventional Southwick-Robinson approach. For exposures beyond T4/T5 a full stenotomy approach is recommended. 4

\section{Summary}

The authors have presented the preferred approach to the cervical spine from Co down to T4. Familiarity with these approaches will allow the surgeon to tackle any pathologies involving the anterior column of the cervical spine. While such approaches are very suited for decompression of the spinal canal, fixation in the form of anterior plating does not afford the same degree of stability compared to posterior segmental fixation. As such, when multiple level fixation and stability is required, such procedures are often combined. Thus anterior approaches to the cervical spine should only be one part of the armamentarium of a spine surgeon, although an important one. 


\section{References}

1. Fang HSY, Ong GB. Direct Anterior Approach to the Upper Cervical Spine. Journal of Bone and Joint Surgery 1962;44:1588-604.

2. Smith GW, Robinson RA. The treatment of certain cervical-spine disorders by anterior removal of the intervertebral disc and interbody fusion. J Bone Joint Surg Am 1958;40-A:607-24.

3. Southwick WO, Robinson RA. Surgical approaches to the vertebral bodies in the cervical and lumbar regions. J Bone Joint Surg Am 1957;39-A:631-44.

4. Luk KD, Cheung KM, Leong JC. Anterior approach to the cervicothoracic junction by unilateral or bilateral manubriotomy. A report of five cases. J Bone Joint Surg Am 2002;84A:1013-7.

5. Shaha AR, Johnson R, Miller J, et al. Transoral-transpharyngeal approach to the upper cervical vertebrae. Am J Surg 1993;166:336-40.

6. Biyani A, An H. Anterior upper cervical spine approaches. In Herkowitz HN ed. The cervical spine surgery atlas. 2nd ed. Philadelphia: Lippincott Williams \& Wilkins, 2003:69

89.

1. Henn JS, Lee MC, Rhoton ALJ. Transoral approach to craniocervical junction and upper cervical spine. In Kim DH, Henn JS, Vaccaro AR, et al. eds. Surgical anatomy \& techniques to the spine. Philadelphia: Saunders Elsevier, 2006:3-32.

2. Winter R, Lonstein J, Dennis F, et al. Anterior upper cervical procedures. Atlas of spine surgery. Philadelphia: W.B. Saunders, 1995:1-17.

3. Crockard H. Midline ventral approaches to the craniocervical junction and upper cervical spine. In Sherk H ed. The cervical spine: an atlas of cervical procedures. Philadelphia: JB Lippincott Co., 1994:93-112.

4. Mendoza N, H.A. C. Anterior transoral procedures. In An HS, Riley LHI eds. An atlas of surgery of the spine. London: Martin Dunitz, 1998:55-69.

5. Robinson RA, Smith G. Anterolateral cervical disk removal and interbody fusion for cervical disk syndrome. Bull Johns Hopkins Hosp 1955;96:223-4.

6. German J, Benzel E, Alexander J. Anatomy and surgical approaches and exposure of the vertebral column, the cervical spine. In Benzel E ed. Spine surgery: technique, complication avoidance and management. New York: Churchill Livingstone, 1999:145-56.

7. Silber J, Albert T. Anterior and anterolateral, mid and lower cervical spine approaches: transverse and longitudinal (C3 to C7). In Herkowitz HN ed. The cervical spine surgery atlas. Philadelphia: Lippincott Williams \& Wilkins, 2003:91-8.

8. Hillard VH, Apfelbaum RI. Surgical management of cervical myelopathy: indications and techniques for multilevel cervical discectomy. Spine J 2006;6:242S-51S.

9. Chang U, Lee MC, Kim DH. Anterior approach to the midcervical spine. In Kim DH, Henn JS, Vaccaro A, et al. eds. Surgical anatomy \& techniques to the spine. Philadelphia: Saunders Elsevier, 2006:45-56.

10. Curylo LJ, Mason HC, Bohlman HH, et al. Tortuous course of the vertebral artery and anterior cervical decompression: a cadaveric and clinical case study. Spine (Phila Pa 1976) 2000;25:2860-4.

11. Birch R, Bonney G, Marshall RW. A surgical approach to the cervicothoracic spine. J Bone Joint Surg Br 1990;72:904-7. 
12. Fang HS, Ong GB, Hodgson AR. Anterior spinal fusion: The operative approaches. Clin Orthop Relat Res 1964;35:16-33.

13. Darling GE, McBroom R, Perrin R. Modified anterior approach to the cervicothoracic junction. Spine (Phila Pa 1976) 1995;20:1519-21. 


\section{Figure legends}

Figure 1. Lateral view of the transoral approach. With the neck in hyperextension and mouth opened wide, the approach generally reaches C0 to C3. A specialized retractor (R) with blades depress the tongue (T) and elevate the soft palate. Sometimes an additional soft tube through the nasal passage (NP) is used to further retract the soft palate.

Figure 2. Specialized oropharyngeal retractor is used to maintain access. The important landmark to identify is the anterior tubercle of C1 (AT) which lies directly under and near the insertion of the anterior longitudinal ligament. The tectorial membrane (TM) is cephalad and the longus coli muscles (LC) flank the anterior longitudinal ligament.

Figure 3. Resection of the dens (D) usually requires burring thin the anterior portion and finally resecting the cortical shell by Kerrison ronguer or an angled curette. The transverse ligament (TL) lie immediately posterior and great care is needed to preserve this to maximize lateral stability.

Figure 4. Anterolateral (Smith-Robinson) approaches using 2 different skin incisions. The transverse skin incision (T) is for up to 2 level surgeries while the longitudinal skin incision

(L) is used for more extensive surgeries.

Figure 5. The Smith-Robinson approach utilizes the internervous plane between the sternocleidomastoid and the deeper longus colli muscles. Our preferred approach for the mid-cervical levels is through a right sided incision, and the longus colli muscles (LC) are retracted to their corresponding sides while the trachea (Tr) and esophagus (Es) are retracted to the left. 
Figure 6. The manubriotomy skin incision is a longitudinal incision that extends from the manubrial notch (MN) to $3 \mathrm{~cm}$ caudad to the sternal angle. The incision may be extended cephalad anterior the the sternocleidomastoid (SCM) if more proximal levels require operating. The medial end of the right clavicle (C) is shown.

Figure 7. The longitudinal osteotomy is made over the midline of the manubrium with the transverse limb cutting across the $2^{\text {nd }}$ intercostal space. Therefore an L-shaped or inverted T-shaped cut is created depending on the width of the approach required.

Figure 8. This schematic diagram illustrates the positions of the main vessels relative to the $3^{\text {rd }}$ thoracic vertebra (T3). On the right the 3thoracic vertebra is flanked by the right brachiocephalic vein (RBv) and artery (A). The left brachiocephalic vein ( $\mathrm{LBv})$ crosses caudal and anterior to the $3^{\text {rd }}$ thoracic vertebra while the left common carotid (LCC) flanks the left. The esophagus and trachea are not shown here as it is retracted to the left under the retractor (TE). 


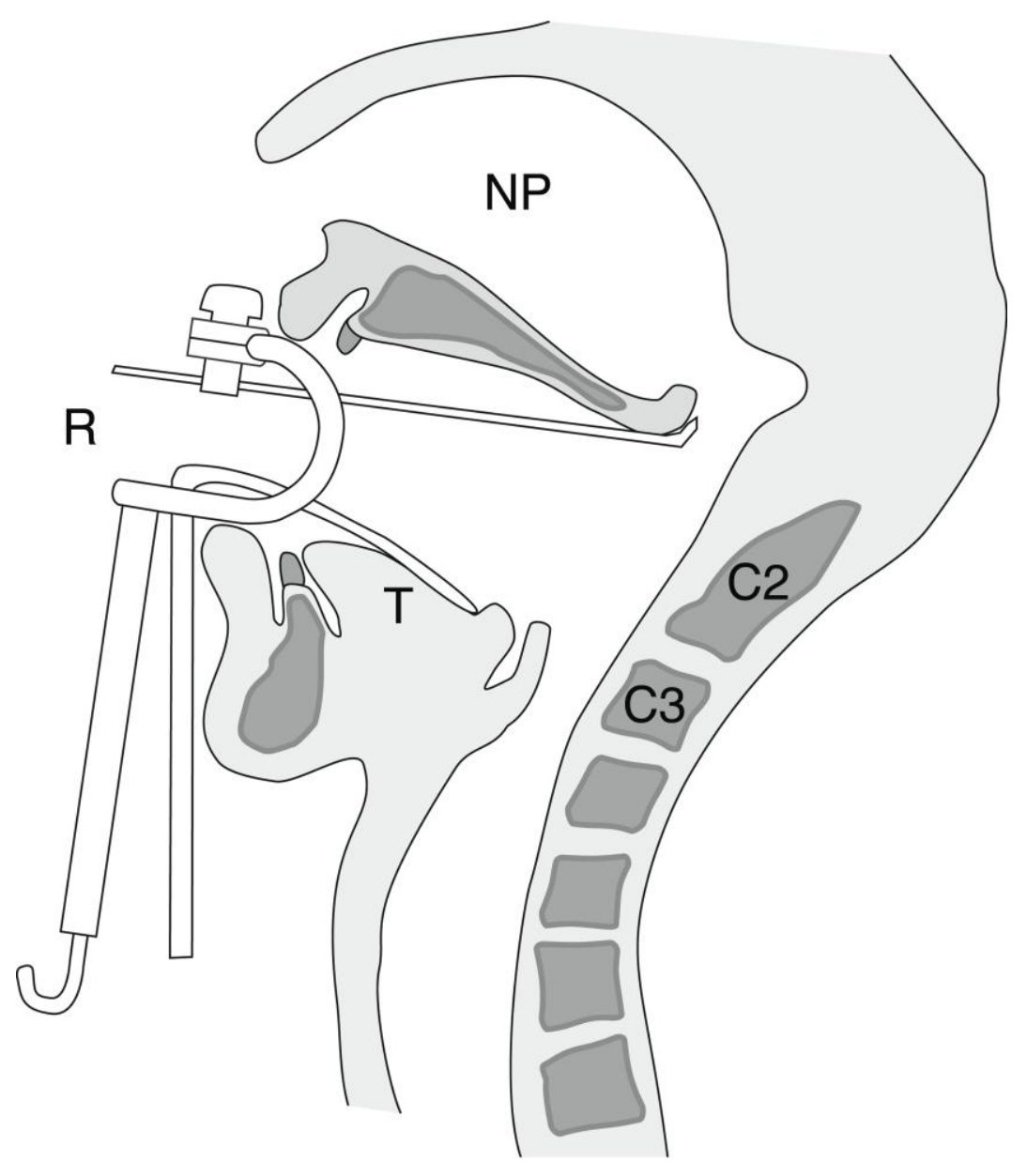

Figures ( TIFF, EPS, or PPT only)

Figure 1. Lateral view of the transoral approach. With the neck in hyperextension and mouth opened wide, the approach generally reaches C0 to C3. A specialized retractor (R) with blades depress the tongue ( $\mathrm{T}$ ) and elevate the soft palate. Sometimes an additional soft tube through the nasal passage (NP) is used to further retract the soft palate. 


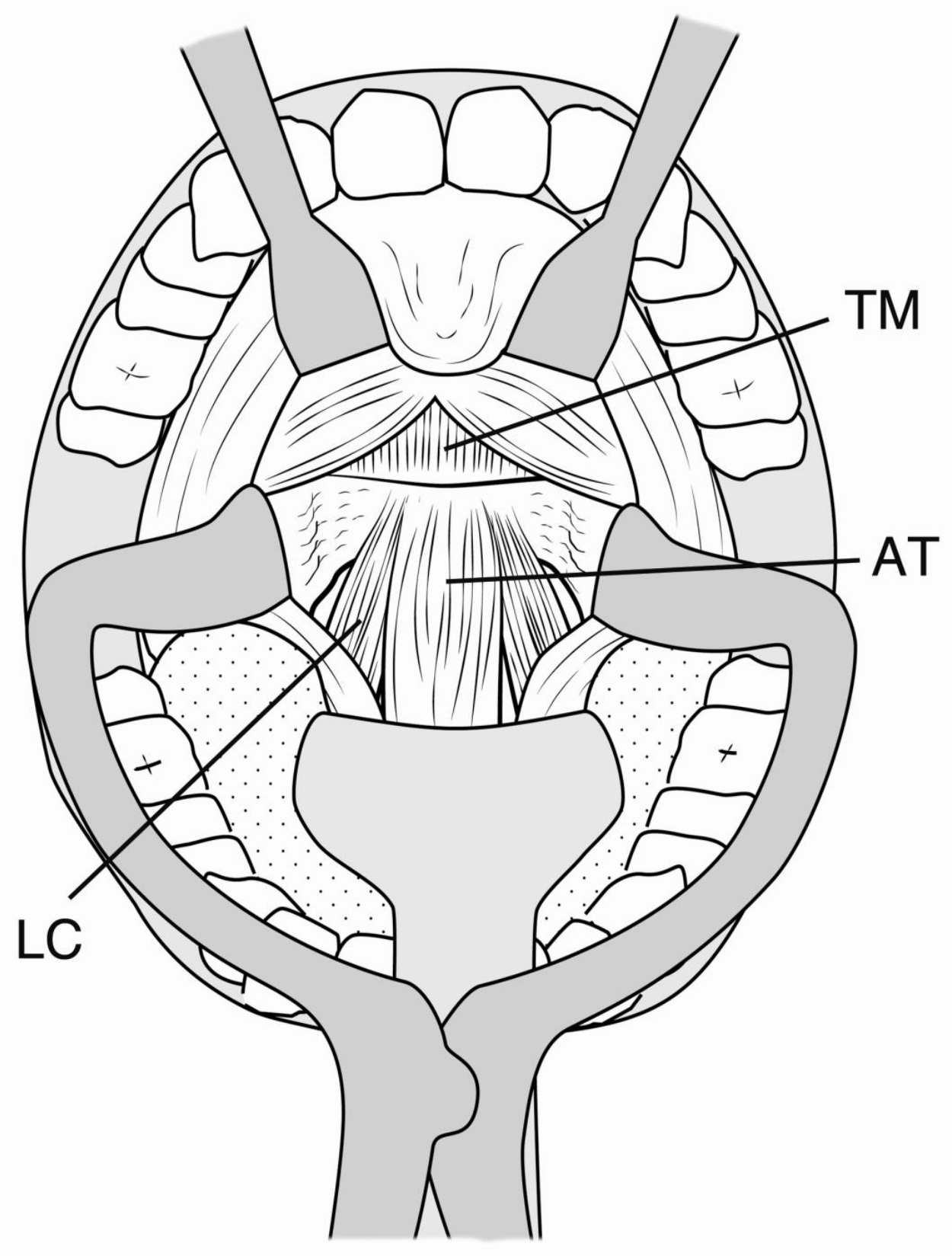

Figure 2. Specialized oropharyngeal retractor is used to maintain access. The important landmark to identify is the anterior tubercle of C1 (AT) which lies directly under and near the insertion of the anterior longitudinal ligament. The tectorial membrane (TM) is cephalad and the longus coli muscles (LC) flank the anterior longitudinal ligament. 


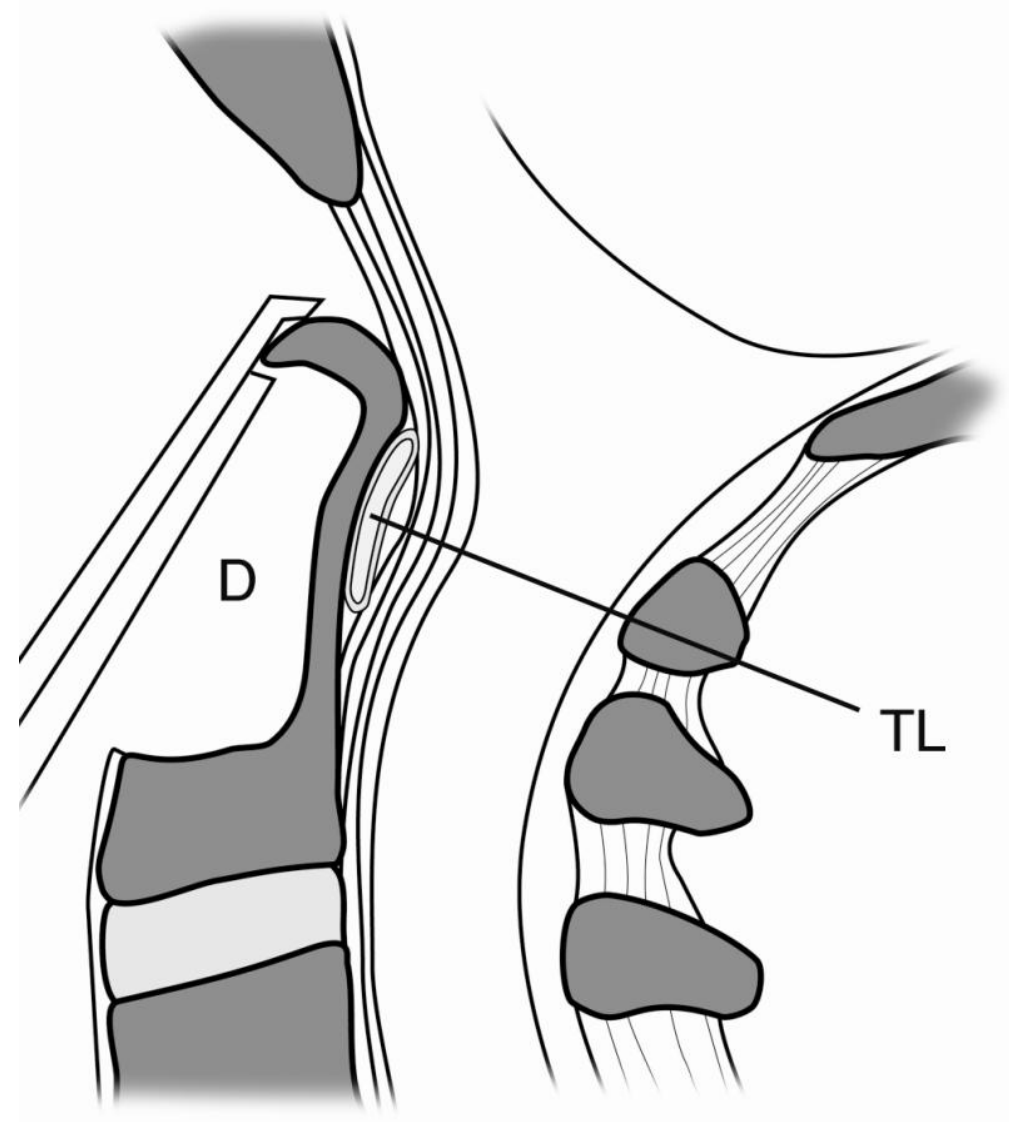

Figure 3. Resection of the dens (D) usually requires burring thin the anterior portion and finally resecting the cortical shell by Kerrison ronguer or an angled curette. The transverse ligament (TL) lie immediately posterior and great care is needed to preserve this to maximize lateral stability. 


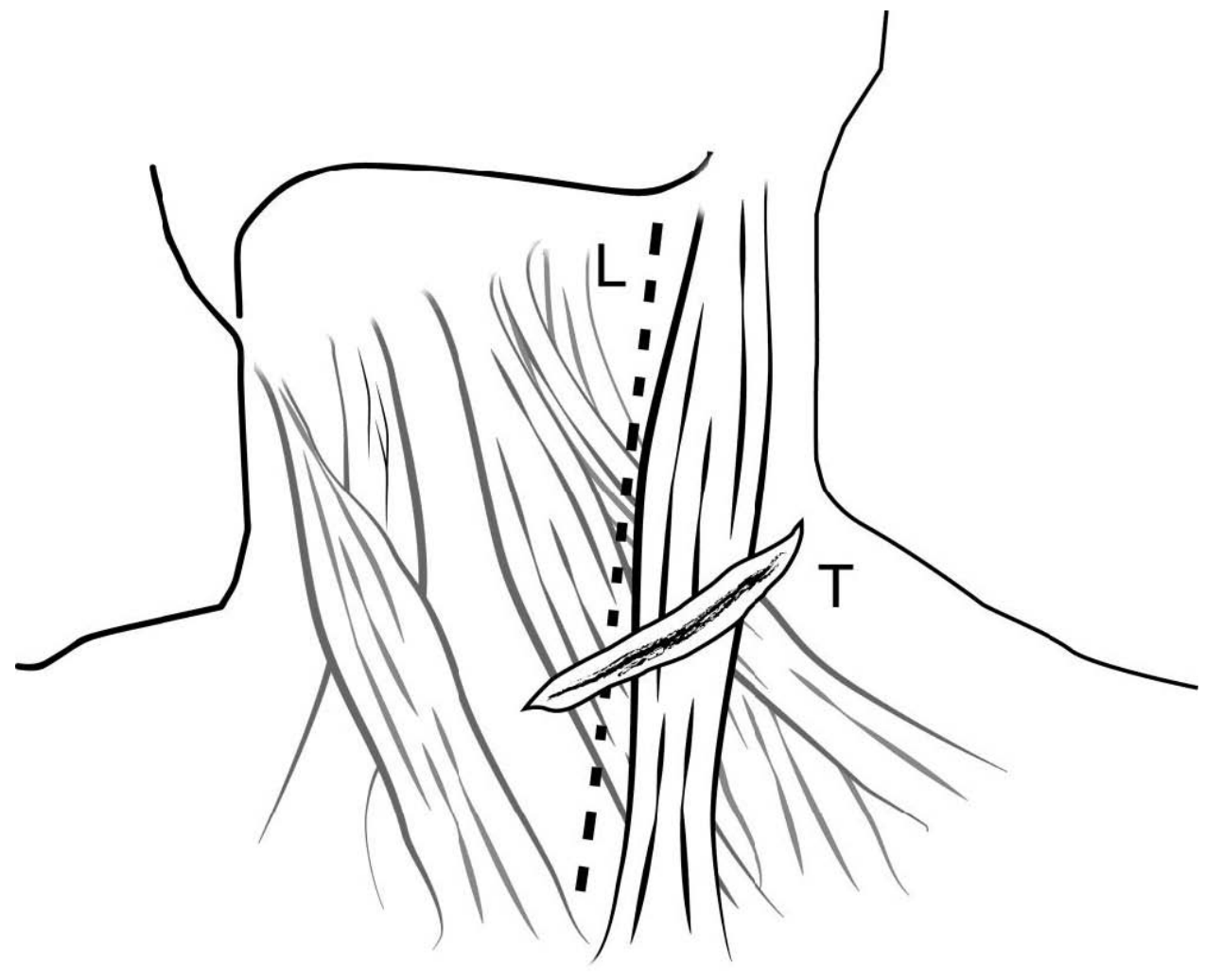

Figure 4.

Anterolateral
(Smith-Robinson)
approaches using 2
different skin
incisions. The
transverse skin
incision (T) is for
up to 2 level
surgeries while the
longitudinal skin

incision

(L) is used for more extensive surgeries. 


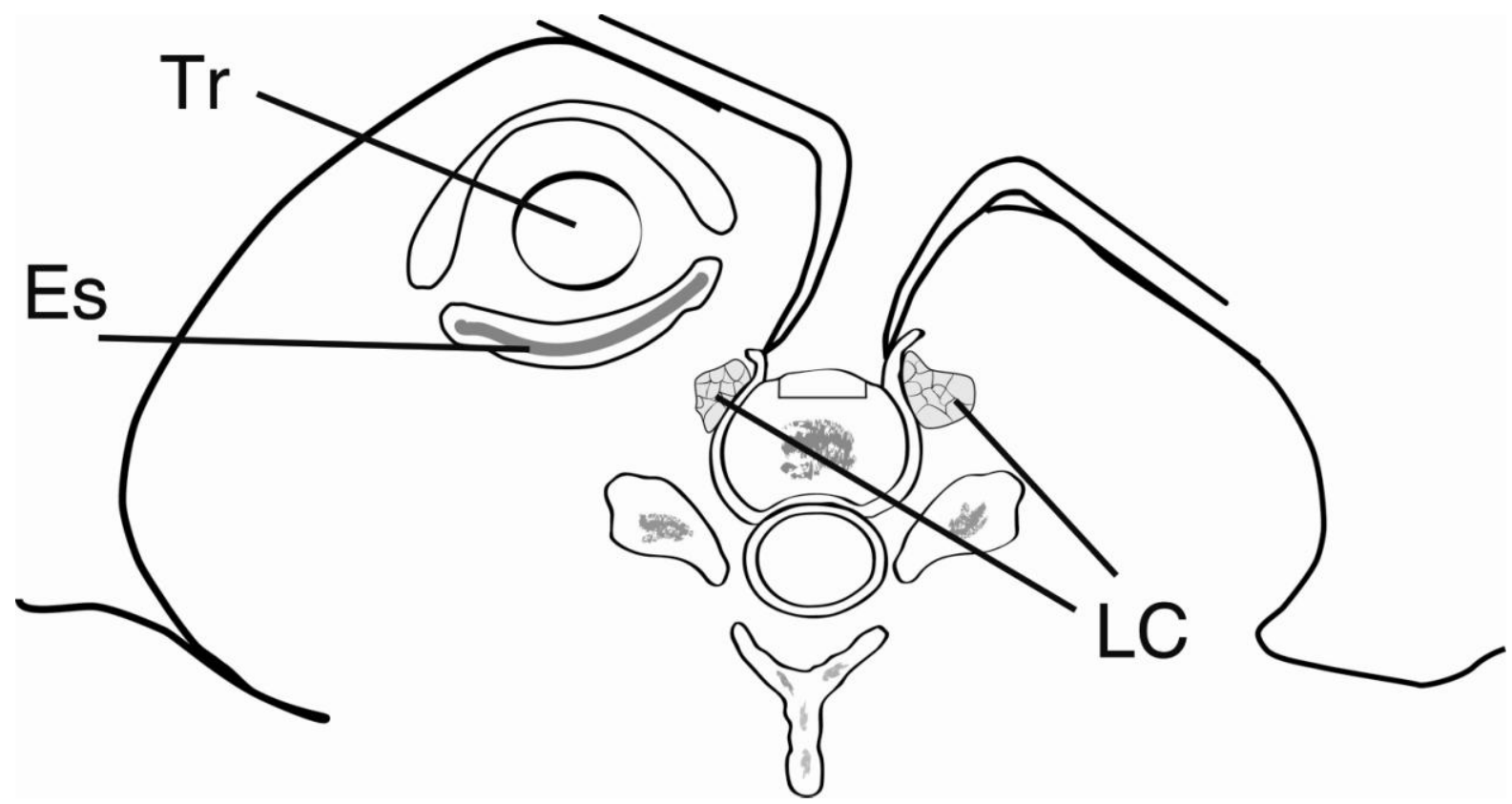

Figure 5. The Smith-Robinson approach utilizes the internervous plane between the sternocleidomastoid and the deeper longus colli muscles. Our preferred approach for the mid-cervical levels is through a right sided incision, and the longus colli muscles (LC) are retracted to their corresponding sides while the trachea (Tr) and esophagus (Es) are retracted to the left. 


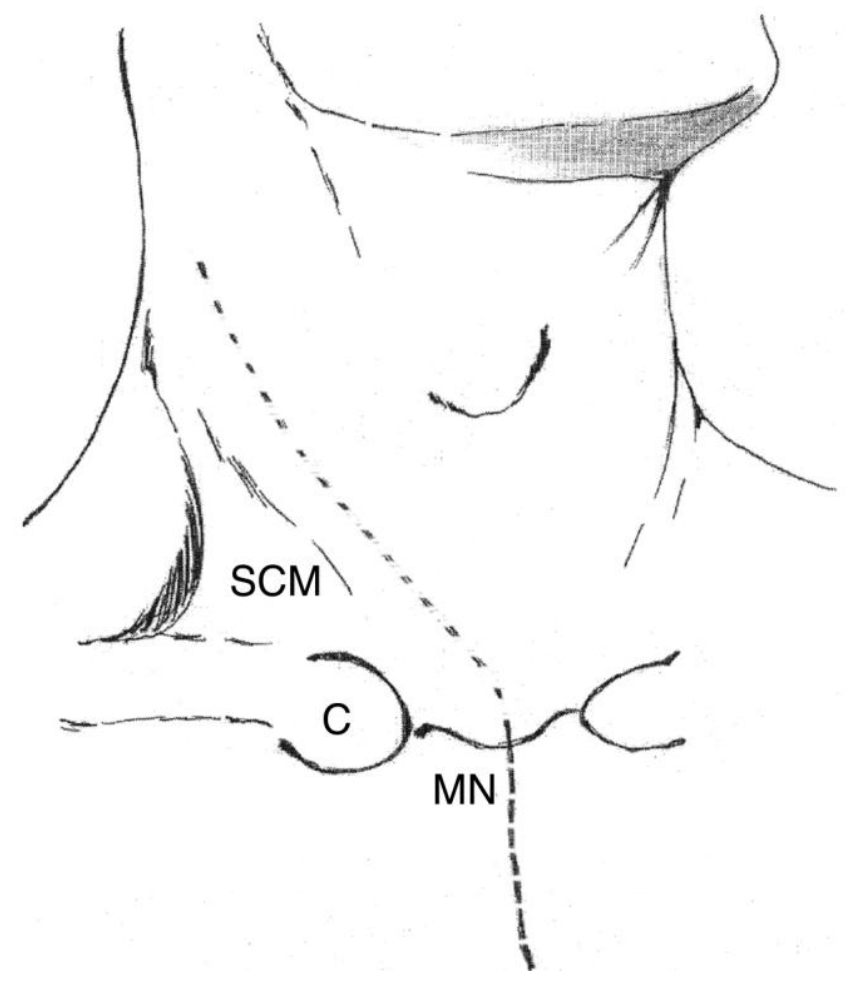

Figure 6. The manubriotomy skin incision is a longitudinal incision that extends from the manubrial notch $(\mathrm{MN})$ to $3 \mathrm{~cm}$ caudad to the sternal angle. The incision may be extended cephalad anterior the the sternocleidomastoid (SCM) if more proximal levels require operating. The medial end of the right clavicle $(\mathrm{C})$ is shown. 


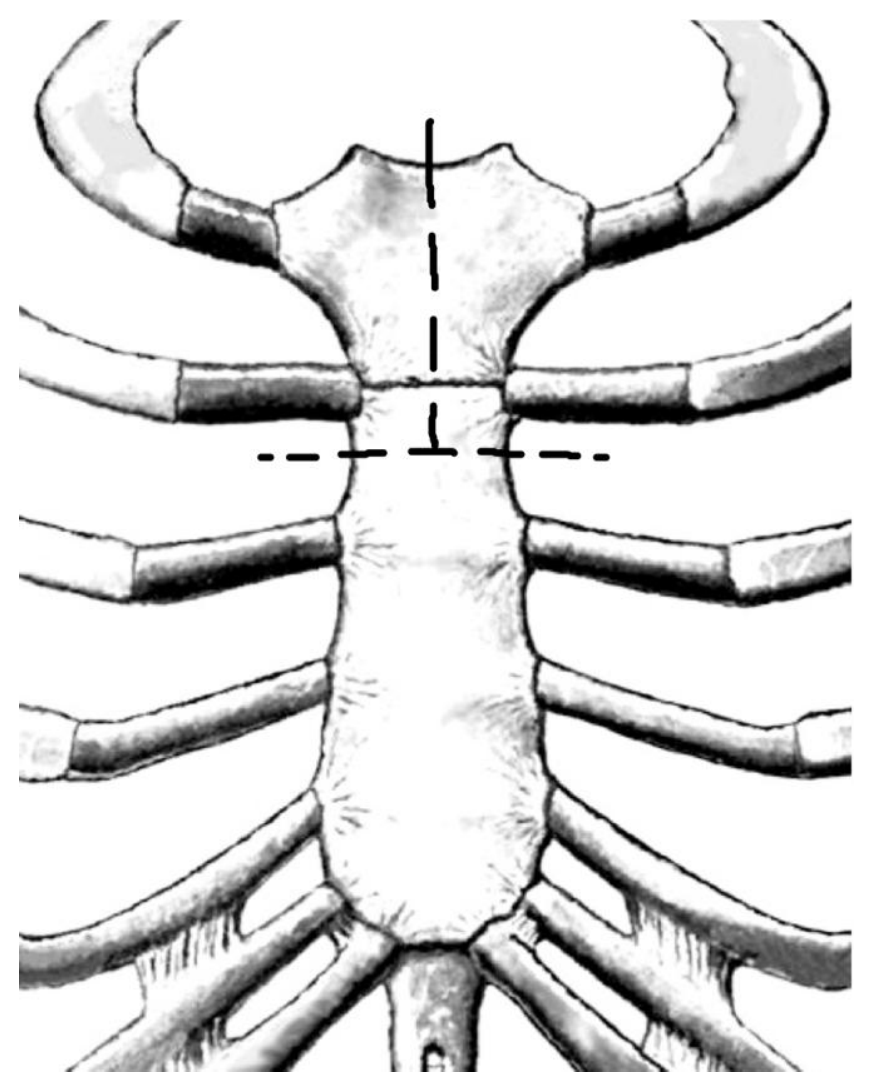

Figure 7. The longitudinal osteotomy is made over the midline of the manubrium with the transverse limb cutting across the $2^{\text {nd }}$ intercostal space. Therefore an L-shaped or inverted T-shaped cut is created depending on the width of the approach required. 


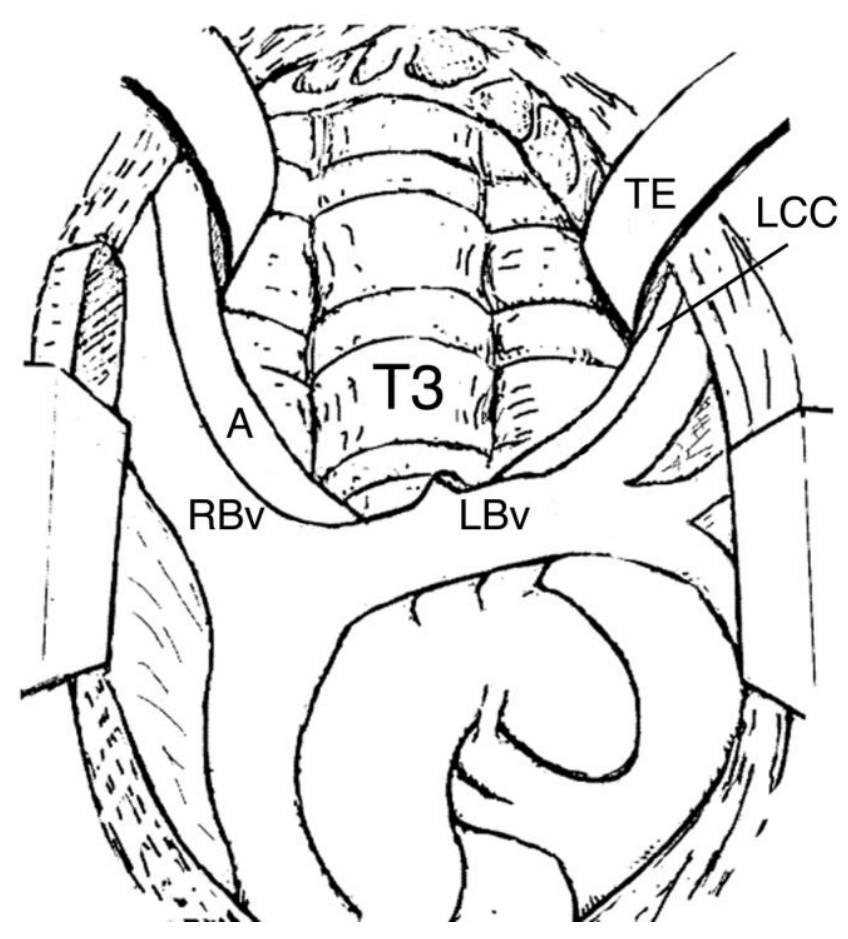

Figure 8. This schematic diagram illustrates the positions of the main vessels relative to the

rd rd

3thoracic vertebra (T3). On the right the 3thoracic vertebra is flanked by the right brachiocephalic vein (RBv) and artery (A). The left brachiocephalic vein (LBv) crosses caudal and anterior to the $3^{\text {rd }}$ thoracic vertebra while the left common carotid (LCC) flanks the left. The esophagus and trachea are not shown here as it is retracted to the left under the retractor (TE). 